\title{
Field effects and ictal synchronization: insights from in homine observations
}

\section{Shennan A. Weiss ${ }^{1}{ }^{*}$, Guy McKhann $\mathrm{Jr}^{2}{ }^{2}$, Robert Goodman ${ }^{2}$, Ronald G. Emerson ${ }^{1}$, Andrew Trevelyan ${ }^{3}$, Marom Bikson ${ }^{4}$ and Catherine A. Schevon ${ }^{1}$}

${ }^{1}$ Department of Neurology, Schevon Lab, Columbia University, New York, NY, USA

${ }^{2}$ Department of Neurosurgery, Columbia University, New York, NY, USA

${ }^{3}$ Department of Neuroscience, Newcastle University, Newcastle, UK

${ }^{4}$ Biomedical Engineering, The City College of The City University of New York, New York, NY, USA

\section{Edited by:}

Hauke R. Heekeren, Freie Universität

Berlin, Germany

Reviewed by:

Mohamad Z. Koubeissi, George

Washington University, USA

F. E. Dudek, University of Utah, USA

*Correspondence:

Shennan A. Weiss, Department of

Neurology, Schevon Lab, Columbia

University, 710 W. 168th Street,

New York, NY 10032, USA

e-mail: saw2164@columbia.edu
It has been well established in animal models that electrical fields generated during inter-ictal and ictal discharges are strong enough in intensity to influence action potential firing threshold and synchronization. We discuss recently published data from microelectrode array recordings of human neocortical seizures and speculate about the possible role of field effects in neuronal synchronization. We have identified two distinct seizure territories that cannot be easily distinguished by traditional EEG analysis. The ictal core exhibits synchronized neuronal burst firing, while the surrounding ictal penumbra exhibits asynchronous and relatively sparse neuronal activity. In the ictal core large amplitude rhythmic ictal discharges produce large electric fields that correspond with highly synchronous neuronal firing. In the penumbra rhythmic ictal discharges are smaller in amplitude, but large enough to influence spike timing, yet neuronal synchrony is not observed. These in homine observations are in accord with decades of animal studies supporting a role of field effects in neuronal synchronization during seizures, yet also highlight how field effects may be negated in the presence of strong synaptic inhibition in the penumbra.

Keywords: ephaptic conduction, field effect, seizures, epilepsy, synchrony
An electrical field effect occurs when currents associated with an extracellular field cross the cell membrane. If the current is significant the transmembrane potential $\left(V_{m}=V_{\text {intracelullar }}-\right.$ $\left.V_{\text {extracellular }}\right)$ will differ from the intracellular potential. If the transmembrane potential surpasses threshold it may initiate firing, or at reduced transmembrane polarization influence action potential timing (Radman et al., 2007a; Anastassiou et al., 2011), synaptic efficacy (Bikson et al., 2004), or other membrane processes (Faber and Korn, 1989; Jefferys, 1995; Weiss and Faber, 2010).

Field effects are thought to play a role in seizure initiation and propagation (Jefferys, 1995; Dudek et al., 1998). In the absence of synaptic transmission, non-synaptic mechanisms are sufficient to initiate and propagate seizure like activity in hippocampal slice models (Jefferys and Haas, 1982; Taylor and Dudek, 1982, 1984; Jiruska et al., 2010). Also, paired extra- and intracellular recordings of spontaneous paroxysmal events in cat neocortex in vivo, with synaptic transmission unaffected, have confirmed that fields associated with ictal discharges depolarize the neuronal membrane and can elicit action potentials (Grenier et al., 2003a,b).

These discharges are thought to be generated by large paroxysmal depolarizing shifts (Goldensohn and Purpura, 1963; Grenier et al., 2003a,b) mediated by glutamatergic synaptic transmission, high-voltage calcium spikes, and a persistent voltage-gated sodium current (Traub et al., 1993). The electric field associated with these currents ranges between 3-9 $\mathrm{mV} / \mathrm{mm}$ (Pockberger et al., 1984; Jefferys, 1995). Early modeling studies found that the electric fields associated with ictal discharges can synchronize action potentials on a time scale of $1 \mathrm{~ms}$ (Traub et al., 1985). Moreover, in hippocampal slices neuronal synchrony during ictal discharges is modulated by changes in osmolality that can strengthen or weaken field effects (Bikson et al., 2003).

The mechanism by which field effects contribute to neural synchronization has been a subject of intense study. It is estimated that DC uniform fields alter the transmembrane potential in individual neurons at the soma (Radman et al., 2009) by $0.18 \mathrm{mV}$ per $\mathrm{mV} / \mathrm{mm}$ field strength (Deans et al., 2007). However, in hippocampal slices bathed in high $\mathrm{K}+$ to elicit epileptiform activity exogenously pulsed uniform fields as small as $295 \mu \mathrm{V} / \mathrm{mm}$ could entrain neuronal firing (Francis et al., 2003). An explanation for the sensitivity of spike timing to weak electric fields may be that network interactions amplify small field effects experienced by all neurons across an extended territory (Parra and Bikson, 2004; Reato et al., 2010; Weiss and Faber, 2010) by modifying the spike timing of a significant portion of the population (Radman et al., 2007b; Anastassiou et al., 2011) and increasing the synchrony of chemical synaptic transmission in an auto-regenerative manner. Recordings from cortical neuronal ensembles, in vitro (Anastassiou et al., 2011), and in vivo (Ozen et al., 2010) confirm 


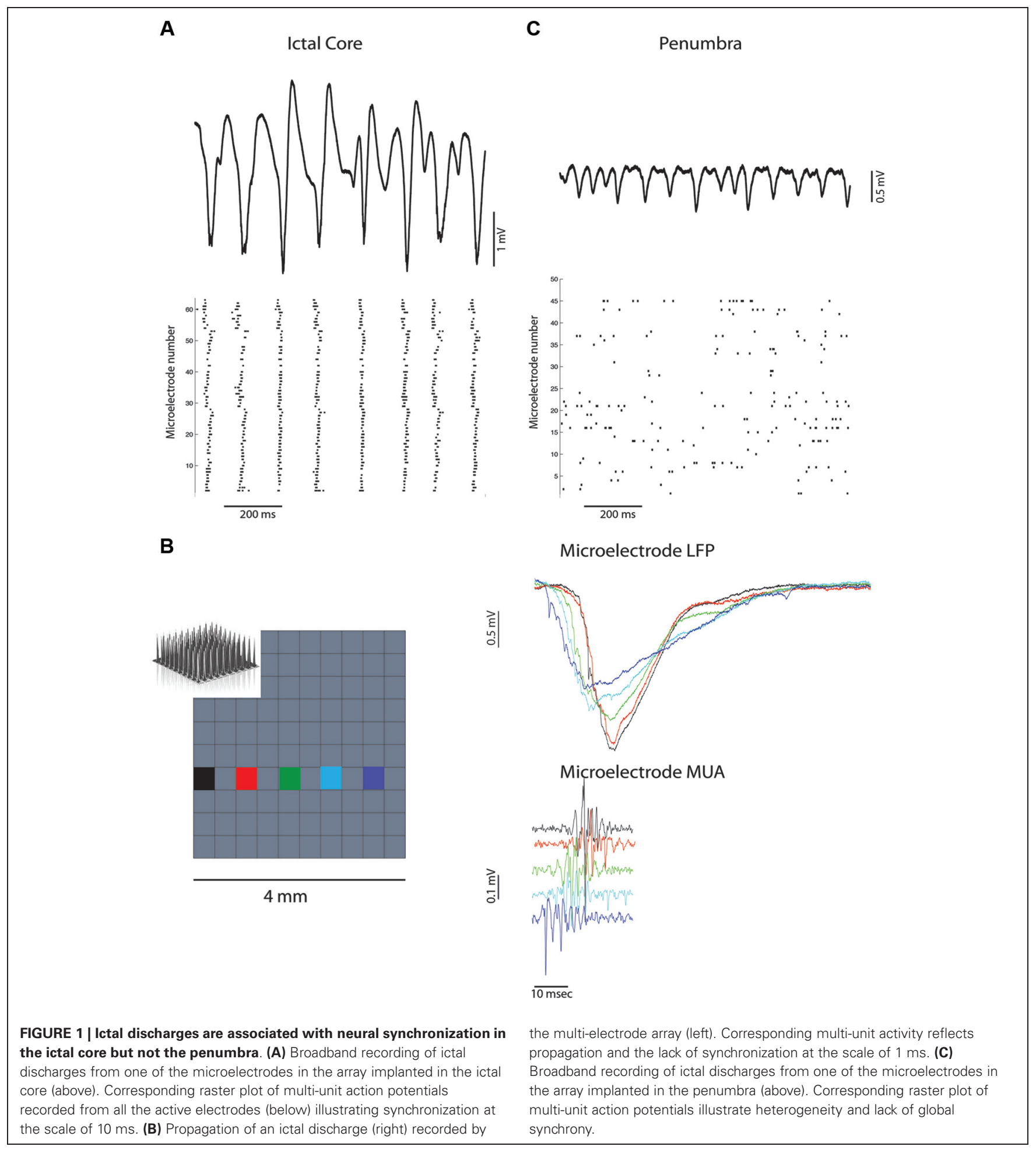

that population level spike coherence to exogenous non-uniform oscillating fields occurs at strengths ranging from $1-4 \mathrm{mV} / \mathrm{mm}$.

If weak electric fields contribute to neuronal synchronization, it would be expected that neuronal synchrony would be observed during the large electric fields generated by ictal discharges in humans. Despite the importance of neural synchrony in seizures, there is a dearth of multi-electrode recordings demonstrating such synchrony over extended cortical territories. Recent recordings of partial seizures from the human cortex with the Utah microelectrode array (House et al., 2006) provide indirect 
evidence both for and against a role for field effects in ictal neural synchronization (Truccolo et al., 2011; Schevon et al., 2012).

Schevon et al., recorded single unit activity during partial seizures with the microelectrode array implanted within the seizure onset zone. In three patients, each of the electrodes detected synchronous unit activity phase locked to the trough of the ictal discharge. However, in two other patients the microelectrode array recorded heterogeneous unit activity (Schevon et al., 2012).

Figure 1A demonstrates marked neural synchrony at the temporal scale of $\sim 10 \mathrm{~ms}$ during ictal discharges when the microelectrode array was implanted in the ictal core. To calculate the electric field strength generated by these ictal discharges requires multi-contact depth electrode recordings. However, a rough estimate can be made using prior depth electrode recordings of ictal discharges induced by penicillin application in rabbit cortex (Pockberger et al., 1984). Based on these recordings, the measured ictal discharge amplitude of 1-2 mV in layer 4/5 corresponds with an electric field with a strength of approximately $2-6 \mathrm{mV} / \mathrm{mm}$. Based on in vitro (Anastassiou et al., 2011), and in vivo (Ozen et al., 2010), evidence this field strength is sufficient to induce population level spike field coherence when the alternating field is applied for an extended duration. Thus, the small variability in the timing of action potentials during ictal discharges suggests that neocortical pyramidal neurons may interact directly via electrical interactions.

Alternatively, neural synchrony during ictal discharges in humans may be solely due to the strong uniform synaptic depolarization and field effects may not play a role. To prove that field effects contribute to neuronal synchronization requires paired intracellular and extracellular recordings from pyramidal neurons during the ictal discharge (Weiss and Faber, 2010). However, paired recordings during ictal discharges recorded from cat neocortex in vivo did demonstrate considerable ephaptic depolarization (Grenier et al., 2003a,b).

Thus, neuronal synchrony during ictal discharges may be enhanced in the ictal core by field effect interactions that synergistically pace and entrain the rhythmic paroxysmal depolarizing shifts generated by glutamatergic synaptic transmission (Traub et al., 1985; Parra and Bikson, 2004). Synchronization at the temporal scale of $\sim 1$ ms does not appear to be achieved over extended territories as ictal discharges propagate across the cortex at speeds of $\sim 500 \mathrm{~mm} / \mathrm{s}$ (Trevelyan et al., 2007; Schevon et al., 2010, 2012), and action potential firing is affected by the lag times (Figure 1B). This does not rule out the possibility of field effects playing a role in synchronization however, since neocortical slow waves which also propagate rapidly across the cortex (Massimini et al., 2004), can produce fields that enhance and entrain network activity locally (Fröhlich and McCormick, 2010).

Figure 1C demonstrates the heterogeneous asynchronous firing during the ictal discharges recorded by the microelectrode array in another patient (Schevon et al., 2012). Similar observations of heterogenous asynchronous firing during human seizures have been previously reported (Truccolo et al., 2011). The microelectrode array was implanted in the ictal penumbra in this case. It is apparent that the ictal discharges are smaller amplitude than that recorded from the ictal core in Figure 1A and produce an estimated electric field across the cortical layers of approximately 1-2.1 $\mathrm{mV} / \mathrm{mm}$ (Pockberger et al., 1984). This field strength should be sufficient to influence spike timing (Francis et al., 2003; Radman et al., 2007a; Anastassiou et al., 2011; Weiss and Faber, 2010), but not necessarily result in strong population level spike field coherence (Ozen et al., 2010; Anastassiou et al., 2011).

Besides a weaker endogenous field, another potential explanation for the heterogeneous, asynchronous firing, in the face of the observed ictal discharges in Figure 1C, is that the neurons in the penumbra have a membrane potential farther from threshold than in the ictal core. Calcium imaging and patch clamp recording from cortical slices bathed in zero magnesium suggest that in the penumbra territory a combination of rhythmic inhibitory postsynaptic potentials (IPSPs) and excitatory post-synaptic potentials (EPSPs) contribute to the ictal discharges (Trevelyan et al., 2006; Trevelyan, 2009; Schevon et al., 2012). Assuming that this is the case in the human ictal penumbra (Figure 1C) the synergistic influence of field effects on neuronal synchronization may be negated. Additional experimental and modeling studies are required to support this hypothesis.

The recordings from the ictal core demonstrate profound neuronal synchrony during ictal discharges. While, paired intra- and extracellular recordings are required to confirm that field effects help to generate this synchronization, the electrical field strength is likely sufficient (Ozen et al., 2010; Anastassiou et al., 2011). In contrast, the recordings from the ictal penumbra highlight how endogenous or exogenous field effects may be affected by synaptic inhibition.

\section{REFERENCES}

Anastassiou, C. A., Perin, R., Markram, H., and Koch, C. (2011). Ephaptic coupling of cortical neurons. Nat. Neurosci. 14, 217-223. doi: 10.1038/nn.2727

Bikson, M., Fox, J. E., and Jefferys, J. G. R. (2003). Neuronal aggregate formation underlies spatiotemporal dynamics of nonsynaptic seizure initiation. J. Neurophysiol. 89, 2330-2333. doi: 10.1152/jn.00764.2002

Bikson, M., Inoue, M., Akiyama, H., Deans, J. K., Fox, J. E., Miyakawa, H., et al. (2004). Effects of uniform extracellular DC electric fields on excitability in rat hippocampal slices in vitro. J. Physiol. 557, 175-190. doi: 10.1113/jphysiol.2003. 055772

Deans, J. K., Powell, A. D., and Jefferys, J. G. R. (2007). Sensitivity of coherent oscillations in rat hippocampus to AC electric fields. J. Physiol. 583, 555-565. doi: 10.1113/jphysiol.2007.137711

Dudek, F. E., Yasumura, T., and Rash, J. E. (1998). "Non-synaptic" mechanisms in seizures and epileptogenesis. Cell Biol. Int. 22, 793-805.

Faber, D. S., and Korn, H. (1989). Electrical field effects: their relevance in central neural networks. Physiol. Rev. 69, 821-863.

Francis, J. T., Gluckman, B. J., and Schiff, S. J. (2003). Sensitivity of neurons to weak electric fields. J. Neurosci. 23, 7255-7261.

Fröhlich, F., and McCormick, D. A. (2010). Endogenous electric fields may guide neocortical network activity. Neuron 67, 129-143. doi: 10.1016/j.neuron.2010. 06.005

Goldensohn, E. S., and Purpura, D. P. (1963). Intracellular potentials of cortical neurons during focal epileptogenic discharges. Science 139, 840-842. doi: 10 . 1126/science.139.3557.840

Grenier, F., Timofeev, I., and Steriade, M. (2003a). Neocortical very fast oscillations (ripples, 80-200 Hz) during seizures: intracellular correlates. J. Neurophysiol. 89, 841-852. doi: 10.1152/jn.00420.2002

Grenier, F., Timofeev, I., Crochet, S., and Steriade, M. (2003b). Spontaneous field potentials influence the activity of neocortical neurons during paroxysmal activities in vivo. Neuroscience 119, 277-291. doi: 10.1016/s0306-4522(03)00101-5

House, P. A., MacDonald, J. D., Tresco, P. A., and Normann, R. A. (2006). Acute microelectrode array implantation into human neocortex: preliminary 
technique and histological considerations. Neurosurg. Focus 20, 1-4. doi: 10. 3171/foc.2006.20.5.5

Jefferys, J. G. (1995). Nonsynaptic modulation of neuronal activity in the brain: electric currents and extracellular ions. Physiol. Rev. 75, 689-723.

Jefferys, J. G., and Haas, H. L. (1982). Synchronized bursting of CA1 hippocampal pyramidal cells in the absence of synaptic transmission. Nature 300, 448-450. doi: $10.1038 / 300448 \mathrm{a} 0$

Jiruska, P., Csicsvari, J., Powell, A. D., Fox, J. E., Chang, W.-C., Vreugdenhil, M., et al. (2010). High-frequency network activity, global increase in neuronal activity and synchrony expansion precede epileptic seizures in vitro. J. Neurosci. 30, 5690-5701. doi: 10.1523/jneurosci.0535-10.2010

Massimini, M., Huber, R., Ferrarelli, F., Hill, S., and Tononi, G. (2004). The sleep slow oscillation as a traveling wave. J. Neurosci. 24, 6862-6870. doi: 10 . 1523/jneurosci.1318-04.2004

Ozen, S., Sirota, A., Belluscio, M. A., Anastassiou, C. A., Stark, E., Koch, C., et al. (2010). Transcranial electric stimulation entrains cortical neuronal populations in rats. J. Neurosci. 30, 11476-11485. doi: 10.1523/jneurosci.5252-09. 2010

Parra, L. C., and Bikson, M. (2004). Model of the effect of extracellular fields on spike time coherence. Conf. Proc. IEEE Eng. Med. Biol. Soc. 6, 4584-4587. doi: 10.1109/IEMBS.2004.1404271

Pockberger, H., Rappelsberger, P., and Petsche, H. (1984). Penicillin-induced epileptic phenomena in the rabbit's neocortex I. The development of interictal spikes after epicortical application of penicillin. Brain Res. 309, 247-260. doi: 10 1016/0006-8993(84)90591-2

Radman, T., Datta, A., and Peterchev, A. V. (2007a). In vitro modulation of endogenous rhythms by AC electric fields: Syncing with clinical brain stimulation. $J$. Physiol. 584, 369-370. doi: 10.1113/jphysiol.2007.140947

Radman, T., Ramos, R. L., Brumberg, J. C., and Bikson, M. (2009). Role of cortical cell type and morphology in subthreshold and suprathreshold uniform electric field stimulation in vitro. Brain Stimul. 2, 215-228, e1-e3. doi: 10.1016/j.brs. 2009.03.007

Radman, T., Su, Y., An, J. H., Parra, L. C., and Bikson, M. (2007b). Spike timing amplifies the effect of electric fields on neurons: implications for endogenous field effects. J. Neurosci. 27, 3030-3036. doi: 10.1523/ineurosci.0095-07. 2007

Reato, D., Rahman, A., Bikson, M., and Parra, L. C. (2010). Low-intensity electrical stimulation affects network dynamics by modulating population rate and spike timing. J. Neurosci. 30, 15067-15079. doi: 10.1523/jneurosci.2059-10.2010

Schevon, C. A., Goodman, R. R., Mckhann, Jr. G., and Emerson, R. G. (2010). Propagation of epileptiform activity on a submillimeter scale. J. Clin. Neurophysiol. 27, 406-411. doi: 10.1097/wnp.0b013e3181fdf8a1

Schevon, C. A., Weiss, S. A., Mckhann, Jr. G., Goodman, R. R., Yuste, R., Emerson, R. G., et al. (2012). Evidence of an inhibitory restraint of seizure activity in humans. Nat. Commun. 3:1060. doi: 10.1038/ncomms2056
Taylor, C. P., and Dudek, F. E. (1982). Synchronous neural afterdischarges in rat hippocampal slices without active chemical synapses. Science 218, 810-812. doi: $10.1126 /$ science.7134978

Taylor, C. P., and Dudek, F. E. (1984). Synchronization without active chemical synapses during hippocampal afterdischarges. J. Neurophysiol. 52, 143-155.

Traub, R. D., Dudek, F. E., Snow, R. W., and Knowles, W. D. (1985). Computer simulations indicate that electrical field effects contribute to the shape of the epileptiform field potential. Neuroscience 15, 947-958. doi: 10.1016/03064522(85) $90245-3$

Traub, R. D., Miles, R., and Jefferys, J. G. (1993). Synaptic and intrinsic conductances shape picrotoxin-induced synchronized after-discharges in the guinea-pig hippocampal slice. J. Physiol. 461, 525-547. doi: 10.1016/03043940(93)90391-w

Trevelyan, A. J. (2009). The direct relationship between inhibitory currents and local field potentials. J. Neurosci. 29, 15299-15307. doi: 10.1523/jneurosci.201909.2009

Trevelyan, A. J., Baldeweg, T., van Drongelen, W., Yuste, R., and Whittington, M. (2007). The source of afterdischarge activity in neocortical tonic-clonic epilepsy. J. Neurosci. 27, 13513-13519. doi: 10.1523/jneurosci.3005-07.2007

Trevelyan, A. J., Sussillo, D., Watson, B. O., and Yuste, R. (2006). Modular propagation of epileptiform activity: evidence for an inhibitory veto in neocortex. $J$. Neurosci. 26, 12447-12455. doi: 10.1523/jneurosci.2787-06.2006

Truccolo, W., Donoghue, J. A., Hochberg, L. R., Eskandar, E. N., Madsen, J. R., Anderson, W. S., et al. (2011). Single-neuron dynamics in human focal epilepsy. Nat. Neurosci. 14, 635-641. doi: 10.1038/nn.2782

Weiss, S. A., and Faber, D. S. (2010). Field effects in the CNS play functional roles. Front. Neural. Circuits 4:15. doi: 10.3389/fncir.2010.00015

Conflict of Interest Statement: The authors declare that the research was conducted in the absence of any commercial or financial relationships that could be construed as a potential conflict of interest.

Received: 07 June 2013; accepted: 17 November 2013; published online: 05 December 2013.

Citation: Weiss SA, McKhann G Jr., Goodman R, Emerson RG, Trevelyan A, Bikson M and Schevon CA (2013) Field effects and ictal synchronization: insights from in homine observations. Front. Hum. Neurosci. 7:828. doi: 10.3389/fnhum.2013.00828

This article was submitted to the journal Frontiers in Human Neuroscience.

Copyright (๑) 2013 Weiss, McKhann, Goodman, Emerson, Trevelyan, Bikson and Schevon. This is an open-access article distributed under the terms of the Creative Commons Attribution License (CC BY). The use, distribution or reproduction in other forums is permitted, provided the original author(s) or licensor are credited and that the original publication in this journal is cited, in accordance with accepted academic practice. No use, distribution or reproduction is permitted which does not comply with these terms. 